\title{
Study on the Instrument Panel Assembly Modal Analysis Basic on CAE Technology
}

\author{
Zhi-Kui MA ${ }^{1, \text { a }}$,Lin $\mathrm{HUA}^{2,3}$,Tian-Wen $\mathrm{SHI}^{2,3}$ \\ ${ }^{1}$ Wuhan University of Technology, School of Materials Science and Engineering, Wuhan 430070, \\ China \\ ${ }^{2}$ Wuhan University of Technology, Hubei Key Laboratory of Advanced Technology for Automotive \\ Components, Wuhan 430070, China \\ ${ }^{3}$ Wuhan University of Technology, School of Automotive Engineering, Wuhan 430070, China
}

Keywords: CAE analysis, Instrument panel, Modal, Frequency.

\begin{abstract}
Modal performance of the automotive instrument panel has been studied in this article. Finite element software Hypersh/ OptiStruct has been used in modal analysis in this article. This paper first calculated the modal frequency and vibration shape of the instrument panel. Engineered modification has been applied to rise the inherent frequency. The result shows this method can effectively optimize the inherent frequency of the instrument panel assembly.
\end{abstract}

\section{Introduction}

The automotive NVH requirement is getting higher and higher with the developing of automotive industry. Vibration of the key components in the car has a high contribution to the NVH performance of the whole vehicle.

Instrument panel (IP), as the largest inner trim parts which directly shown to passengers, have not only beauty and function requires but also NVH and safety request $[1,2]$. The vibration and noise on dashboard can be directly feel by passengers. Thus the modal analysis of instrument panel plays an important role in NVH character of the whole vehicle[3].

CAE analysis is a very efficient way to study the NVH character. As it can help shorten the test cycle and reduce development cost, CAE analysis is widely used during automotive development procedure $[4,5]$. Kim M.S. etc. studied the dynamic impact of passenger air-bag(PAB) module by using FEM to predict the dynamic characteristics of vehicle ride safety against head impact[6].Wang P etc. did a impact simulation tests for head and knee bolster as well as NVH analysis[7]. Yang S.M etc. simulated the screw loosening mechanism of a fastening screw used on automotive door trim parts. Clamping force is suggested through the analysis result of reduction according to the tightening torque [8].

In this article, finite-element model (FE model) of each instrument panel part is first set up based on 3D model in Hypermesh. After that, the complete FE model is created with FE parts assembled together and boundary conditions added on it. After that, the modal result is carried out by Hypermesh/ OptiStruct. Then some engineered modification is applied to the instrument panel design to improve its modal character.

\section{Modelling and Boundary Condition}

Modelling: An instrument panel developed for a multi-purpose vehicle is taken into modal analysis. Hypermesh is used to create the FE model. Fig.1 shows the3D model of the instrument panel assembly. The FE model is set up shown as fig. 2 with shell element. It contains 241000 quads and 15000 triangle elements. 


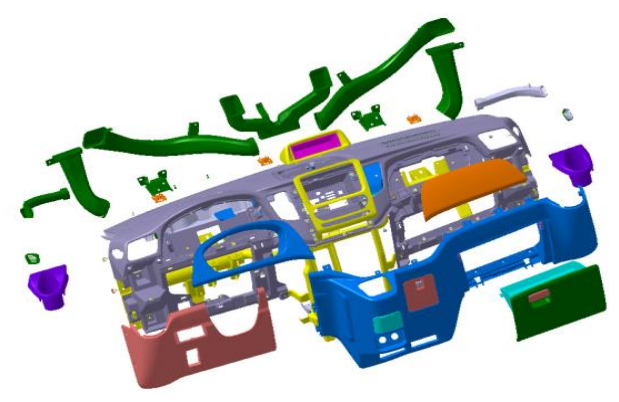

Fig 1. Instrument panel assembly model

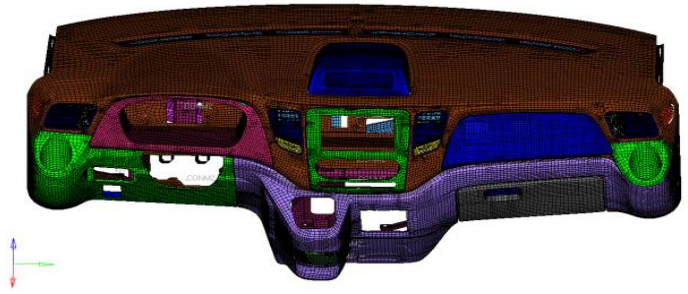

Fig2. Finite element model

Some necessary simplifications are made to help made the analysis more efficiency. Mass points with centroid coordinates are used to instead of air bag, media player system, instrument, air condition and steering system. Bolt joint are simplified as REB2 rigid link which is shown as fig 3.

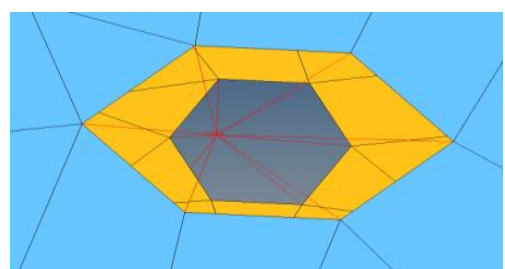

Fig 3. REB 2 connection

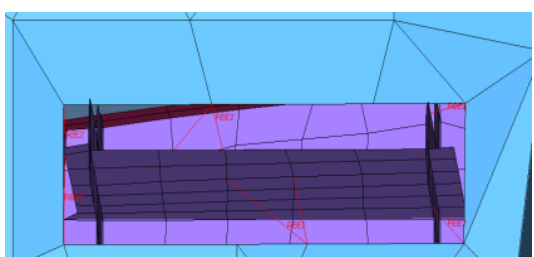

Fig 4.Rigid connection

Most of the components are connected through buckles. These buckles are treated as rigid connected which is shown in Fig 4.

Materials: Most of the instrument parts are made by PP-EPDM-T20.But there are also some other materials used in this instrument panel assembly. Four air outlet, glove box handle, center media control panel as well as instrument cover are made by PC/ABS. AC duct are made by HDPE. The CCB, of course, is made by steel. Materials properties are shown in Tab 1.

Tab1. Materials property

\begin{tabular}{|l|l|l|l|}
\hline Materials & Young 's modulus (MPa) & Poisson's ratio & Density(g/cm3) \\
\hline PP-EPDM-T20 & 1906 & 0.415 & 1.1 \\
\hline PC/ABS & 2300 & 0.4 & 1.1 \\
\hline HDPE & 1000 & 0.426 & 0.85 \\
\hline Steel & 210000 & 0.3 & 7.8 \\
\hline
\end{tabular}

Boundary conditions:The instrument panel is installed on CCB while CCB is fixed at both ends. At the same time, there are three steel buckles in front of IP to fix the IP onto car body. These constrains are shown in Fig 5. Fixed constraint are applied on these nodes to fix all six degrees of freedom In CAE software.

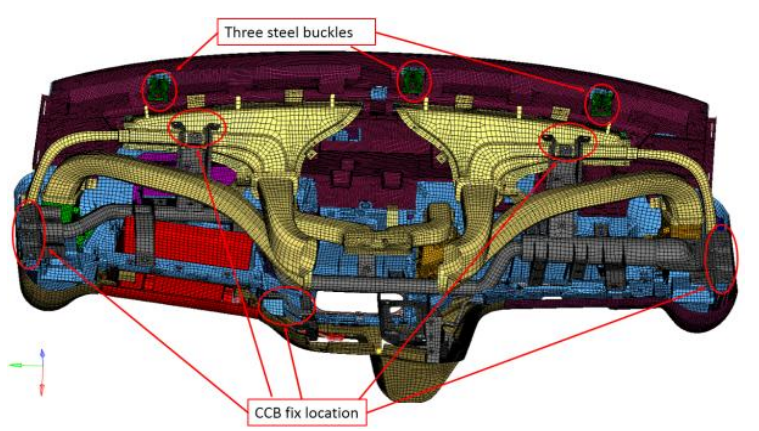

Fig 5. Constrains 


\section{Modal Analysis Request and Method}

The enginein this vehicle has four cylinder. The idle frequency of a four cylinder engine is $25 \mathrm{HZ}$.

Automotive components are usually designed to have different inherent frequency in order not to resonate with engine. For instrument pane, its inherent frequency should be designed higher than $25 \mathrm{HZ}$.

Modal analysis is a technique used to calculate the vibration shapes and associated frequencies that a structure will exhibit. The equilibrium equation for a structure performing free vibration appears as the eigenvalue problem:

$$
[K-\lambda M] x=0
$$

Where, $\mathrm{K}$ is the stiffness matrix of the structure and $\mathrm{M}$ is the mass matrix. Damping is neglected in this calculation. The solution of the eigenvalue problem yields $n$ eigenvalues $\lambda$, where $n$ is the number of degrees-of-freedom. The vector is the eigenvector corresponding to the eigenvalue. The natural frequency fi follows directly from the eigenvalue $\sqrt{\lambda_{i}}$.

$$
f_{i}=\frac{\sqrt{\lambda_{i}}}{2 \pi}
$$

The eigenvalue problem is solved using Lanczos. Simulation job is done by Hypermesh/OptiStruct.

\section{Results}

Modal results are ready from the result file. Tab2 shows the first six orders of modal. The vibration shapes are shown in Fig 6.

Tab 2. Modal frequency

\begin{tabular}{|l|l|}
\hline Order & Frequency $(\mathbf{H z})$ \\
\hline 1 & 23.5 \\
\hline 2 & 30.3 \\
\hline 3 & 46.0 \\
\hline 4 & 50.8 \\
\hline 5 & 54.5 \\
\hline 6 & 55.7 \\
\hline
\end{tabular}

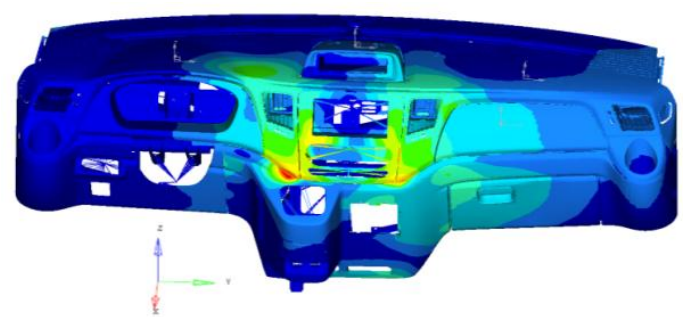

(1) First order frequency vibration shape

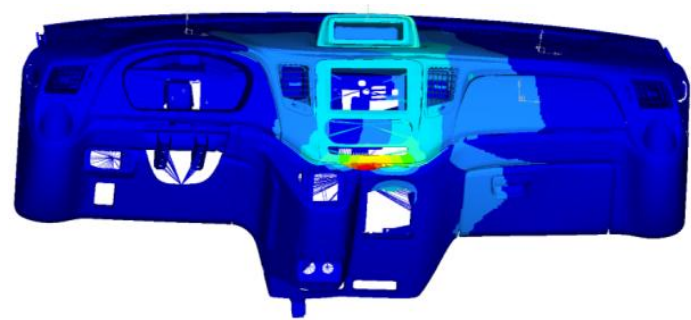

(2) second order frequency vibration shape

Fig 6. Vibration shape

From the result, the first order modal is $23.5 \mathrm{HZ}$. The passenger side lower panel and center media panel will twist along $\mathrm{z}$ direction on that frequency. The second order modal is $30.3 \mathrm{HZ}$. The center media panel frame will bend toward its normal direction.

In can be seen from the vibration shape that the vibration of the assembly will occur on the passenger side lower panel and center media panel connection area. Which means this area is a 
weak area that easy to cause resonance. Some engineered modification work is done to help the first order frequency of the instrument panel larger than $25 \mathrm{~Hz}$.

One is to add two connections between passenger side lower panel and instrument body by buckles as shown if Fig 7. This will connect the passenger side lower panel and instrument body tighter.

Another modification is to add reinforce ribs under passenger side lower panel at the weak area as shown in Fig 8. This will increase the local stiffness on that weak area and help change the natural frequency of the component to avoid resonance.

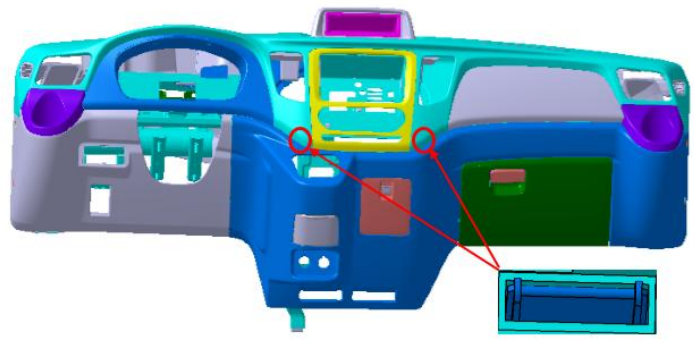

Fig 7. Buckles adding location.

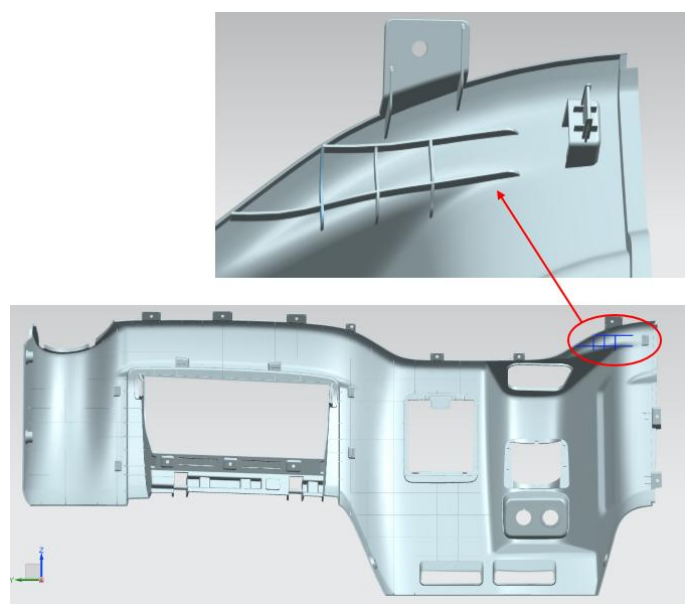

Fig 8. Ribs adding location.

FE model is rebuild and modal is recalculated by Hypermesh/OptStruct again after these modification. The vibration frequency is shown in Tab 3. The vibration shape is shown in Fig 9.

Tab 3. Modal frequency

\begin{tabular}{|l|l|}
\hline Order & Frequency $(\mathbf{H z})$ \\
\hline 1 & 26.5 \\
\hline 2 & 34.5 \\
\hline 3 & 46.6 \\
\hline 4 & 51.5 \\
\hline 5 & 54.7 \\
\hline 6 & 55.8 \\
\hline
\end{tabular}

The first order vibration frequency rise from $23.5 \mathrm{~Hz}$ to $26.5 \mathrm{~Hz}$ after modification which meet the design requirement. The first two orders frequency in this design have a significant increase compare with the original modal.

From the vibration shape photograph shown in Fig 9, the passenger side lower panel and center media panel will twist along $\mathrm{z}$ direction on $26.5 \mathrm{~Hz}$. The second order modal frequency is $34.5 \mathrm{HZ}$. The center media panel frame will bend toward its normal direction. The third order modal frequency is $46.6 \mathrm{HZ}$. Glove box assembly will shake in and out on that frequency. The forth order modal frequency is $51.5 \mathrm{HZ}$. AC duct will shake on that direction. The fifth order modal frequency is 54.7HZ.Glove box body will bend towards $\mathrm{X}$ direction. The sixth order modal frequency is $55.8 \mathrm{HZ}$. The edge of driver side instrument panel will bend toward $\mathrm{z}$ direction. 


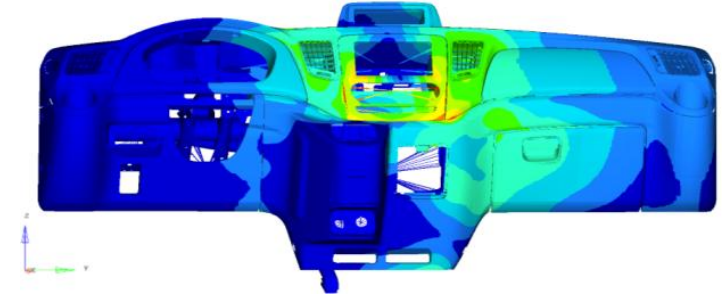

(1) First order frequency vibration shape

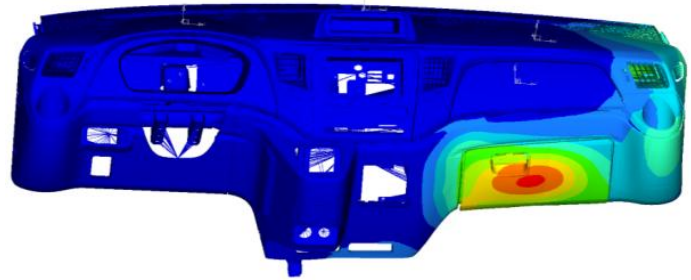

(3) Third order frequency vibration shape

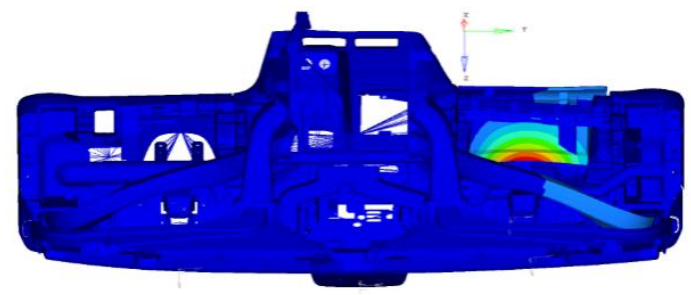

(5) Fifth order frequency vibration shape

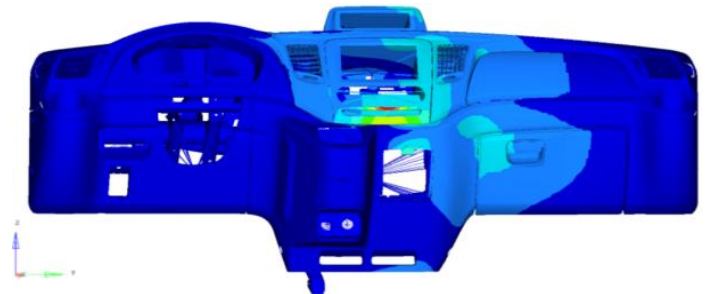

(2) Second order frequency vibration shape

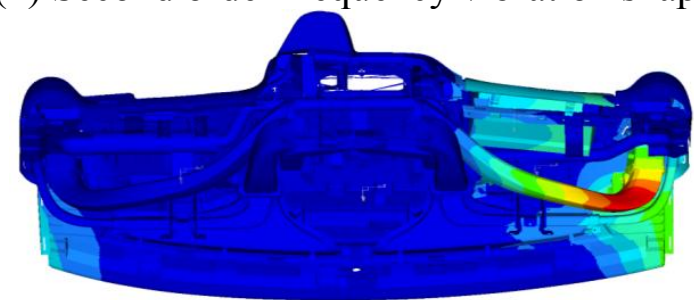

(4) Forth order frequency vibration shape

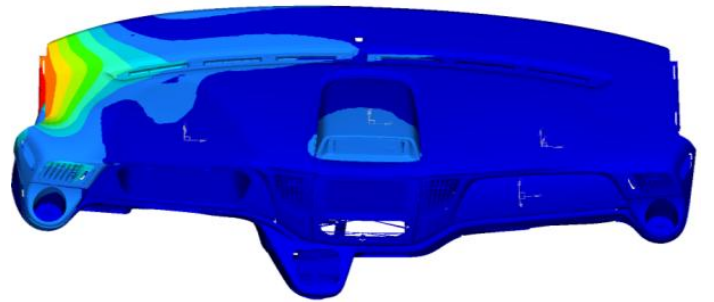

(6) Sixth order frequency vibration shape

Fig 9. Vibration shape

\section{Conclusion}

Modal analysis have beendone in this study. Modal frequency has been calculated and vibration shape has been carried out in this paper. Engineered modification has been made on instrument panel modal to avoid resonate with engine. The result shows this modification can rise the inherent frequency effectively.

A modal analysis method basic on finite element software is also given in this paper. This method can save time and improve efficiency during automotive components development process.

\section{References}

1. ZHOU T, FAN X, TIAN G, et al. Experimental analysis of nonlinear vibration property of instrument panel [J]. Jixie Gongcheng Xuebao/Chinese Journal of Mechanical Engineering, 2008, 44(8): 40-4.

2. ZHAO T, CHAI L. Automotive instrument panel design and manufacturer [J]. Bus technology and research, 2010, 6(3.

3. LI G, DENG T, LU R. Modal analysis and structure optimization of vehicle instrument panel [J]. Automobile applied technology, 2016, 5(3.

4. LI S. Analysis of Instrument Panel Vibration of a Certain Type Aircraft [J]. Trainer, 2012,

5. XIA H, YING X U, ZHANG Y. Numerical Simulation and Experimental Analysis of Roadway Surrounding Rock Loose Circle under Blasting Vibration [J]. 2013,

6. KIM M S, LEE J H, MOON B Y. Dynamic analysis of head impact test of passenger air-bag module assembly of vehicle using FEM [J]. Int J Mod Phys B, 2008, 22(9-11): 1699-704.

7. WANG P, WU G Q. Multidisciplinary Design Optimization of Vehicle Instrument Panel Based on Multi-objective Genetic Algorithm [J]. Chin J Mech Eng, 2013, 26(2): 304-12. 
8. YANG S M, YOO S H, GI D S, et al. LOOSENING ANALYSIS FOR FASTENING SCREW OF AUTOMOTIVE DOOR TRIM PARTS [J]. Int J Automot Technol, 2016, 17(4): 671-9. 